\title{
Incidence and complications of cannula changes in long-term tracheotomized patients: a prospective observational study
}

\author{
Jan Wiefhoff ${ }^{1} \cdot$ Oliver Jansen ${ }^{1} \cdot$ Oliver Kamp $\mathbb{1}^{2} \cdot$ Mirko Aach $^{3} \cdot$ Thomas A. Schildhauer $^{1} \cdot$ Christian Waydhas $^{1}$ • \\ Uwe Hamsen $\mathbb{1}^{1}$
}

Received: 8 March 2019 / Revised: 24 June 2019 / Accepted: 26 June 2019 / Published online: 16 July 2019

(c) The Author(s), under exclusive licence to International Spinal Cord Society 2019

\begin{abstract}
Study design Prospective, monocentric observational study.

Objective Investigation of incidence and complication rate of cannula changes in long-term tracheotomized patients suffering spinal cord injury.

Setting University hospital in Bochum, Germany.

Methods Prospective data collection of all cannula changes between September 2016 and September 2017. Physicians recorded mechanical complications and techniques to solve them, and/or complications resulting in patient-threatening condition.

Results There were 149 cannula changes during 3191 observation days. Overall, urgent cannula changes occurred 2.1 times per 100 observation days. Within the first 8 weeks after tracheostomy, urgent cannula changes were necessary four times per 100 observation days, and were mandatory less than two times per 100 observation days thereafter. Overall, mechanical complications occurred in $12 \%$ of cannula changes, and $8 \%$ of cannula changes were accompanied by patient-threatening complications. Accidental decannulation (AD) occurred in 0.97 of 100 observation days. Recannulation after AD was accompanied by $29 \%$ of mechanical complications during reinsertion, and $16 \%$ led to patient-threatening complications. The major risk factors for mechanical complications were the time lag between cannula change and tracheostomy, and the urgency of the procedure while the thyroid cartilage-jugular distance was significantly associated with patient-threatening complications.

Conclusion $\mathrm{AD}$ and the requirement for urgent cannula changes are common and often related with mechanical and patientthreatening complications. Even weeks after tracheostomy, caregivers need to be aware of serious events, and therefore provide monitoring, knowledge, and appropriate resources to handle these events.
\end{abstract}

\section{Introduction}

Tracheostomy is a common procedure in long-term ventilated and/or neurologically impaired patients [1], especially in spinal cord injury (SCI) [2]. The primary indications

Uwe Hamsen

uwe.hamsen@bergmannsheil.de

1 Department of General and Trauma Surgery, BG University Hospital Bergmannsheil, Bochum, Germany

2 Department of Trauma Surgery, University Hospital Essen, Essen, Germany

3 Department of Spinal Cord Injuries, BG University Hospital Bergmannsheil, Bochum, Germany for tracheostomy are upper airway obstruction to facilitate weaning from mechanical ventilation, secure management of the airway, and for clearance of bronchial secretion [1].

Extensive research has focused on the optimal timing of tracheostomy during the course of the intensive care unit (ICU) treatment, procedure-related complications, and ICU outcomes, such as weaning from ventilation and ventilatorassociated pneumonia. Less research investigated about the outcome after ICU discharge [3, 4].

Interprofessional tracheostomy teams to care about tracheotomized patients have been established and proposed by some authors $[5,6]$. It has been reported that a care by an intensivist-led multidisciplinary team is associated with shorter cannulation periods and length of stay [6]. A standardized care provided by an interprofessional 
Table 1 The demographic data, body dimensions, and number and timing of cannula changes

\begin{tabular}{llll}
\hline & Total $(n=146)$ & OST $(n=31)$ & PDT $(n=115)$ \\
\hline Number of patients $(n)$ & 49 & 17 & 32 \\
Male/female $(n)$ & $40 / 9$ & $14 / 3$ & $26 / 6$ \\
Age (median (IQR)) & $64(54-72)$ & $70(63-75)$ & $61(39-69)$ \\
Days after tracheostomy at study inclusion & $28(0-117)$ & $69(27-202)$ & $14(0-104)$ \\
(median (IQR)) & & & \\
Body height, mean \pm SD (cm) & $177 \pm 10$ & $178 \pm 7$ & $176 \pm 11$ \\
Body weight, mean \pm SD (kg) & $81 \pm 19$ & $78 \pm 15$ & $83 \pm 21$ \\
Neck circumference, mean \pm SD $(\mathrm{cm})$ & $42.7 \pm 6$ & $42.6 \pm 7$ & $42.8 \pm 5$ \\
$\begin{array}{l}\text { Distance between thyroid cartilage and jugulum, mean } \pm \\
\text { SD (cm) }\end{array}$ & $6.5 \pm 1$ & $6.2 \pm 1$ & $6.7 \pm 1$ \\
Duration of observation per patient (median (IQR)) & $50(22-101)$ & $61(26-103)$ & $45(20-97)$ \\
Total days of observation $(n)$ & 3191 & 1238 & 1953 \\
Patients discharged decannulated $(n(\%))$ & $31(63)$ & $11(65)$ & $20(63)$ \\
Cannula changes $(n)$ & 146 & 31 & 115 \\
Median days between cannula changes $(\mathrm{IQR})$ & $7(2-15)$ & $17(6-49)$ & $7(1-13)$ \\
\hline
\end{tabular}

$O S T$ open surgical tracheostomy, $P D T$ percutaneous dilatational tracheostomy, $I Q R$ interquartile range, $S D$ standard deviation

tracheostomy team resulted in fewer tracheostomy-related complications and an increased use of speaking valves [5]. However, a systematic review and meta-analysis concluded that there is only weak evidence with regard to tracheostomy teams reducing total tracheostomy time and increasing the use of speaking valves [7].

Severe complications may occur, including respiratory failure and death due to the tracheostomy tube occlusion $[5,6,8]$ or accidental decannulation (AD) [9]. The incidence of $\mathrm{AD}$ and complications associated with elective or urgent cannula changes are not well known [10].

This prospective observational study was performed in order to determine the incidence of cannula changes, associated mechanical and patient-related complications, as well as to identify risk factors related to these complications.

\section{Methods}

This prospective, monocentric observational study was performed at the Department of General and Trauma Surgery and the Department of Spinal Cord Injuries, BG University Hospital Bergmannsheil, Bochum, Germany. It has been approved by the local ethics committee (no. of approval 16-5889-BR). During a 12-month period from September 2016 to September 2017, all admitted patients with SCI with a tracheostomy tube in place or tracheotomized at our site were enrolled into the study after obtaining informed consent. Patients were either treated on intensive and intermediate care unit (IMC) or normal ward.

All cannula changes were performed by physicians. Each physician is experienced in the care for spinal cord injured patients, either presently working in the ICU or with at least 12-month full-time qualification in intensive care medicine. No standardized protocol regarding indication or technique for cannula changes or decannulation was implemented, and therapy depended on bedside decision of physician on duty.

Possible complications were divided into mechanical complications or complications resulting in patientthreatening conditions.

Mechanical complications were defined as subjective and/or objective problems (as judged by the operator) in reestablishing the airway. Recannulation against resistance, impossibility to recannulate with a cannula of the same size, needs for oral intubation or redilatation of the tracheostomy canal were documented.

Complications resulting in a potential threat of the patient included bleeding leading to either surgical intervention and/or blood transfusion, soft tissue emphysema, pneumothorax, injuries of the trachea, and abnormal vital signs with respect to hemodynamic or respiratory deterioration during cannula change. Respiratory deterioration was defined as an oxygen saturation below 90\%. Hemodynamic deterioration was defined as tachycardia above 120 beats per min, bradycardia below 60 beats per min, hypotension below $90 \mathrm{mmHg}$ systolic blood pressure. minor bleeding. Major bleeding was defined as bleeding leading to either surgical intervention and/or blood transfusion, intermediate bleeding leading to aspirable blood during bronchoscopy, while minor bleeding could be handled without intervention beyond compression or sealing.

For later analysis, minor bleeding was excluded from statistical analysis, as it is considered as not relevant.

We measured patients body weight and height, in addition neck circumference was measured at the insertion point
Bleeding was subdivided into major, intermediate, and 
Table 2 Complications during cannula changes

\begin{tabular}{|c|c|c|c|}
\hline & Total $(n=146)$ & $\operatorname{OST}(n=31)$ & PDT $(n=115)$ \\
\hline Exchanges with mechanical complications, $n$ ( $\%$ of all exchanges) & $18(12)$ & $1(3)$ & $17(15)$ \\
\hline Patients without mechanical complications during cannula change & 25 & & \\
\hline Mechanical complications per patient, $n$ & 0.37 & 0.06 & 0.53 \\
\hline Mechanical complications per 100 observation days, $n$ & 0.56 & 0.08 & 0.87 \\
\hline $\begin{array}{l}\text { Resistance, overcome with same cannula size, } n \text { (\% of all exchanges with mechanical } \\
\text { complications) }\end{array}$ & $11(61)$ & 0 & $11(65)$ \\
\hline $\begin{array}{l}\text { Resistance, overcome with smaller cannula size without resistance, } n \text { (\% of all exchanges with } \\
\text { mechanical complications) }\end{array}$ & $3(17)$ & 0 & $3(18)$ \\
\hline $\begin{array}{l}\text { Resistance, overcome with smaller cannula size against resistance, } n \text { (\% of all exchanges with } \\
\text { mechanical complications) }\end{array}$ & $3(17)$ & $1(100)$ & $2(4)$ \\
\hline Resistance, recannulation not possible, $n$ ( $\%$ of all exchanges with mechanical complications) & $1(6)$ & 0 & $1(6)$ \\
\hline Exchanges with patient-related complications, $n$ ( $\%$ of all exchanges) & $11(8)$ & 0 & $11(10)$ \\
\hline Patients without patient-related complications during cannula change, $n$ & 30 & & \\
\hline Patient-related complications per patient, $n$ & 0.22 & 0 & 0.34 \\
\hline Patient-related complications per 100 observation days, $n$ & 0.34 & 0 & 0.56 \\
\hline Exchanges with respiratory disturbance ${ }^{\mathrm{a}}, n$ ( $\%$ of patient-related complications) & $11(100)$ & 0 & $11(100)$ \\
\hline Desaturation, $n$ & 10 & 0 & 10 \\
\hline Airway obstruction, $n$ & 3 & 0 & 3 \\
\hline Exchanges with cardiocirculatory disorders ${ }^{\mathrm{a}}, n$ ( $\%$ of patient-related complications) & $3(27)$ & 0 & $3(27)$ \\
\hline Tachycardia, $n$ & 1 & 0 & 1 \\
\hline Bradycardia, $n$ & 2 & 0 & 2 \\
\hline Hypotension, $n$ & 1 & 0 & 1 \\
\hline Atropine administered, $n$ & 1 & 0 & 1 \\
\hline Cardiac massage, $n$ & 1 & 0 & 1 \\
\hline
\end{tabular}

${ }^{a}$ Reasons for disturbance may be more than one of those below

of the tracheostomy and distance between thyroid cartilage and jugulum (t.-j. distance) was defined as measured distance between the incisura jugularis sterni and the prominentia laryngea in a supine position using a flexible tape measure.

\section{Statistic}

Statistical analysis was performed using Excel (Microsoft ${ }^{\circledast}$ Excel $^{\circledast}$ 2010, Redmont, WA, USA), and Rstudio (RStudio Version $1.1 .383^{\odot} 2009-2017$ RStudio, Inc, Boston, MA, USA). The data are presented as mean, standard deviation (SD), and median, where appropriate. Multivariate logistic regression was performed using Generalized Linear Models (glm; [binomial]), and backward selection (stepAIC; backward) $[11,12]$ to determine independent risk factors for mechanical and patient-threatening complications.

\section{Results}

Within the 12-month period, 49 tracheotomized patients were included in the study. Forty of them were male (82\%).
Median age (IQR) was 64 (54-72) years. Tetraplegia was present in 35 patients, and paraplegia was present in 14 patients.

Percutaneous dilatational tracheostomy (PDT) had been performed in 32 patients, while 17 patients had received open surgical tracheostomy (OST). Thirty-one (63\%) of 49 initially cannulated patients were successfully decannulated during hospital stay (65\% with OST and 63\% with PDT). No patient died during hospital stay.

The median (IQR) observation period per patient was 50 (22-102) days, as the total number of observed days with tracheostomy was 3191 days. Within these 3191 days, 146 cannula changes were done (4.58 cannula changes per 100 observation days (OD)).

Table 1 shows demographic data, body measurements, and numbers and timing of cannula changes.

Mechanical complications occurred in $18(12 \%)$ cannula changes. Patient-threatening complications during cannula changes occurred in $11(8 \%)$ cases. Not all exchanges with mechanical complications resulted in patient-threatening complications and vice versa. No soft tissue emphysema, tracheal injuries, pneumothorax, or the need for oral intubation associated with a cannula change occurred. 
Table 3 Indication and urgency of cannula changes

\begin{tabular}{|c|c|c|c|c|}
\hline & & Total & OST & PDT \\
\hline $\begin{array}{l}\text { Elective change, } n \text { (\% of all } \\
\text { exchanges) }\end{array}$ & & $79(54)$ & $23(74)$ & $56(49)$ \\
\hline \multirow{11}{*}{$\begin{array}{l}\text { Urgent change, } n \text { ( } \% \text { of all } \\
\text { exchanges) }\end{array}$} & & $67(46)$ & $8(26)$ & $59(51)$ \\
\hline & Intratracheal misplacement of cannula, $n$ & 2 & 0 & 2 \\
\hline & $\begin{array}{l}\text { Intraluminal obstruction of cannula by } \\
\text { secretions, } n\end{array}$ & 22 & 4 & 18 \\
\hline & Others, $n$ & 12 & 2 & 8 \\
\hline & $\begin{array}{l}\text { Accidental decannulation, } n \text { ( } \% \text { of all } \\
\text { exchanges) }\end{array}$ & $31(21)$ & $1(3)$ & $30(26)$ \\
\hline & Incomplete dislocation (soft tissue), $n$ & 7 & 0 & 7 \\
\hline & Complete dislocation, $n$ & 24 & 1 & 23 \\
\hline & Self-decannulation, $n$ & 12 & 1 & 11 \\
\hline & Decannulation during mobilization, $n$ & 5 & 0 & 5 \\
\hline & Decannulation through coughing, $n$ & 2 & 0 & 2 \\
\hline & $\begin{array}{l}\text { Unknown reason for accidental } \\
\text { decanulation, } n\end{array}$ & 12 & 0 & 12 \\
\hline
\end{tabular}

OST open surgical tracheostomy, PDT percutaneous dilatational tracheostomy

Table 4 Urgency of cannula changes correlated to complications

\begin{tabular}{|c|c|c|c|c|}
\hline & & Elective & Urgent (without AD) & Urgent for $\mathrm{AD}$ \\
\hline$n$ & & 79 & 36 & 31 \\
\hline \multirow{5}{*}{$\begin{array}{l}\text { Exchanges with mechanical } \\
\text { complication, } n(\% *)\end{array}$} & & $5(6)$ & $4(11)$ & $9(29)$ \\
\hline & Resistance, overcome with same cannula size, $n$ & 3 & 4 & 4 \\
\hline & $\begin{array}{l}\text { Resistance, overcome with smaller cannula size } \\
\text { without resistance, } n\end{array}$ & 1 & 0 & 2 \\
\hline & $\begin{array}{l}\text { Resistance, overcome with smaller cannula size } \\
\text { against resistance, } n\end{array}$ & 1 & 0 & 2 \\
\hline & No recannulation, $n$ & 0 & 0 & 1 \\
\hline \multirow{3}{*}{$\begin{array}{l}\text { Exchanges with patient-related } \\
\text { complication, } n(\% *)\end{array}$} & & $2(3)$ & $4(11)$ & $5(16)$ \\
\hline & Respiratory, $n$ & 2 & 4 & 5 \\
\hline & Cardiocirculatory, $n$ & 1 & 1 & 1 \\
\hline
\end{tabular}

$\mathrm{AD}$ accidental decannulation

*Percentage calculated as $\%$ of all exchanges of the same category (elective/urgent without AD/urgent for AD)

Table 2 shows the data on mechanical and patientthreatening complications.

Twenty-seven (18\%) cannula changes were technically supported by using either a tube exchanger $(21,14 \%)$ or a bronchoscope $(5,3 \%)$. Of those 27 changes using technical support, 21 (17 tube exchanger, 4 bronchoscope) were without mechanical complications, while 6 had mechanical complications. In 3 of 27 changes using technical support, a patient-threatening complication occurred. No statistical correlation could be revealed between using technical support and mechanical complications (odds ratio (OR) 2.5; 95\% CI: $0.70-8.32 ; p=0.1)$ or patient-related complications (OR 1.73; 95\% CI: 0.28-7.90; $p=0.42$ ).
Table 3 shows data on cannula changes with respect to indication and urgency.

In all, $46 \%$ of all changes were considered as urgent cases. The main reason for urgent cannula change was $\mathrm{AD}$ (31 out of 67 urgent cannula changes (46\%)).

Table 4 shows data with a closer look at mechanical and patient-threatening complications regarding urgency and indication of change.

Logistic regression and backward selection $[11,12]$ were performed to determine risk factors for mechanical and patient-associated complications (Table 5).

Patient-threatening complications occurred more often during urgent cannula changes than in elective cannula 
Table 5 Logistic regression and backward selection for mechanical (a) and patientthreatening (b) complications

\begin{tabular}{|c|c|c|c|c|c|c|}
\hline & \multicolumn{3}{|c|}{ Logistic regression } & \multicolumn{3}{|c|}{ After backward selection } \\
\hline & OR & $95 \% \mathrm{CI}$ & $p$ & OR & $95 \% \mathrm{CI}$ & $p$ \\
\hline \multicolumn{7}{|c|}{ (a) Mechanical complication } \\
\hline Male & 1 & & & & & \\
\hline Female & 2.16 & $0.18-25.74$ & 0.535 & & & \\
\hline Days after tracheostomy & 0.99 & $0.97-1.00$ & 0.074 & 0.98 & $0.97-1.00$ & 0.022 \\
\hline OST & 1 & & & & & \\
\hline PDT & 3.27 & $0.35-105.52$ & 0.390 & & & \\
\hline Elective change & 1 & & & 1 & & \\
\hline Urgent change & 7.82 & $1.74-54.83$ & 0.016 & 7.50 & $1.96-41.74$ & 0.008 \\
\hline Age & 0.93 & $0.88-0.98$ & 0.014 & 0.94 & $0.90-0.99$ & 0.011 \\
\hline Body height & 0.99 & $0.89-1.10$ & 0.854 & & & \\
\hline Body weight & 0.94 & $0.88-1.00$ & 0.051 & 0.94 & $0.87-0.99$ & 0.040 \\
\hline Neck circumference & 1.40 & $1.11-1.82$ & 0.006 & 1.35 & $1.08-1.72$ & 0.009 \\
\hline T.J. distance & 0.88 & $0.44-1.75$ & 0.712 & & & \\
\hline \multicolumn{7}{|c|}{ (b) Life-threatening complication } \\
\hline Male & 1 & & & & & \\
\hline Female & 5.83 & $0.19-268.87$ & 0.333 & & & \\
\hline Days after tracheostomy & 1.00 & $0.99-1.02$ & 0.636 & & & \\
\hline OST & 1 & & & 1 & & \\
\hline PDT & $\mathrm{n} / \mathrm{a}$ & $\mathrm{n} / \mathrm{a}$ & $\mathrm{n} / \mathrm{a}$ & $\mathrm{n} / \mathrm{a}$ & $\mathrm{n} / \mathrm{a}$ & $\mathrm{n} / \mathrm{a}$ \\
\hline Elective change & 1 & & & 1 & & \\
\hline Urgent change & 3.75 & $0.73-32.45$ & 0.154 & 4.86 & $1.11-34.25$ & 0.058 \\
\hline Age & 0.96 & $0.87-1.07$ & 0.369 & & & \\
\hline Body height & 1.18 & $0.96-1.66$ & 0.212 & & & \\
\hline Body weight & 0.87 & $0.70-0.98$ & 0.058 & 0.89 & $0.77-0.98$ & 0.056 \\
\hline Neck circumference & 1.48 & $1.01-2.79$ & 0.090 & & & \\
\hline T.J. distance & 0.21 & $0.01-1.03$ & 0.133 & 1.5 & $1.12-2.25$ & 0.017 \\
\hline
\end{tabular}

OST open surgical tracheostomy, $P D T$ percutaneous dilatational tracheostomy, $T J$ thyroid cartilageJugulum, $n / a$ not applicable, $O R$ odds ratio, $C I$ confidence intervall

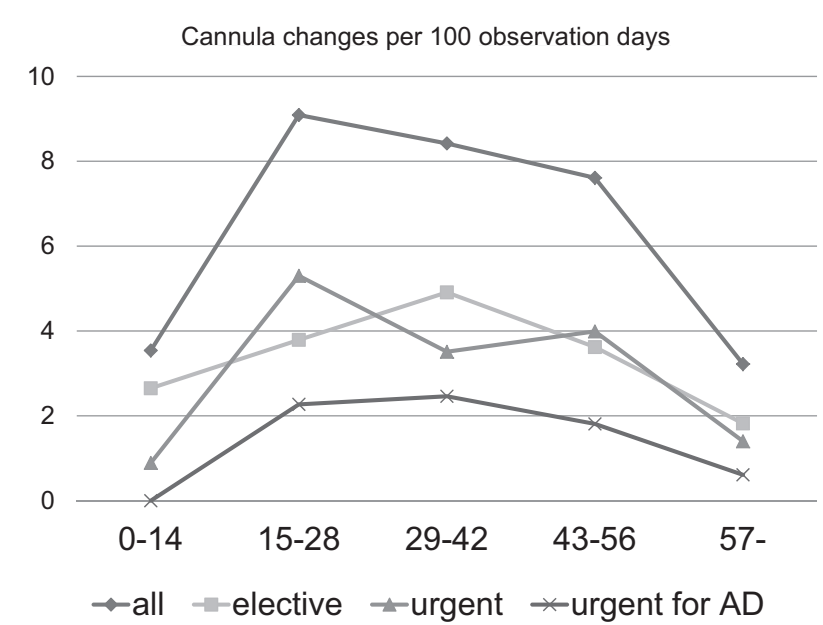

Fig. 1 Incidence of cannula changes depending of days after tracheostomy

changes ( 9 out of 67 urgent changes vs. 2 out of 79 elective changes, $13 \%$ vs. $3 \%$, respectively, OR $5.91 ; 95 \% \mathrm{CI}$ : $1.11-58.15, p=0.02)$.

Mechanical complications occurred significantly more often during urgent cannula changes compared with elective changes (13/67 vs. $5 / 79,19 \%$ vs. $6 \%$, respectively, OR 3.53, 95\% CI: $1.10-13.44, p=0.02$ ).

Figure 1 shows incidence and urgency of cannula changes as a function of time lag after tracheostomy.

Within the first 2 weeks after tracheostomy, 3.54 cannula changes per 100 OD were observed, and no AD occurred. Within the second 2 week after tracheostomy, the incidence of elective and urgent cannula changes increased (9.09 per 100 OD overall, 3.79 per 100 OD elective, 5.30 per 100 OD urgent). Thereafter, the overall incidence of cannula changes decreased. Only for exchanges after week 8 , the incidence of elective and urgent cannula changes decreased to below the incidence of the first 2 weeks.

\section{Discussion}

In this prospective observational study of long-term tracheotomized patients with SCI, we were able to ascertain that mechanical and patient-associated complications during the reinsertion of dislocated cannulas or the exchange of those are common, even weeks after tracheostomy. To our knowledge, no prior study analyzed the incidence of 
cannula changes and procedure-related complication rates in a long-term setting.

We determined an incidence of cannula changes of 4.58 per 100 observation days, and an incidence of urgent changes of 2.1 per 100 observation days. Mechanical complications occurred in 0.56 per 100 observation days. The rate of patient-associated complications during cannula changes was 0.34 per 100 observation days.

White et al. [9] observed a rate of $0.42 \pm 0.09$ of $\mathrm{AD}$ in long-term tracheotomized patients per 100 tracheostomy days. After implementation of a series of interventions, including staff education and increased availability of telemetry and oximetry, they were able to significantly reduce the rate to $0.27 \pm 0.19$ per 100 tracheostomy days. In our study, we observed an incidence of 0.97 AD per 100 observation days, which is about twice as high as the one reported by White et al. Their study was conducted in a long-term acute care hospital, a respiratory step-down unit, and the authors state that they are not sure whether their findings would be generalizable for acute care hospitals. They did neither report on complications related to these urgent cannula changes due to $\mathrm{AD}$ nor the incidence of cannula changes due to other reasons than AD.

Our study revealed an incidence of 0.97 per 100 observation days, which is twice as high as reported by White. We also showed that incidences of AD decreases 6 weeks after tracheostomy. White et al. did not report on mean time since tracheostomy of their study population, but we assumed that their patients were even longer tracheotomized and that our findings complement to their findings taking a closer look on acute care hospitals.

Tabaee et al. [13] published a study on practice patterns and safety for tracheotomy tube change. They performed a survey of chief residents in otolaryngology-head and neck surgery programs in the United States. First tube change was performed after a mean of 5.3 days (range 3-7) after the initial procedure. In all, $42 \%$ of responders reported awareness of a loss of airway and 15\% awareness of a death as a result of a first cannula change at their institution during their residency. The authors discuss their results as surprisingly high and as an unacceptable rate of major complications. No information was provided about complication rates of consecutive tube changes. Another study reported on first cannula change in a retrospective analysis of 151 pediatric patients [14]. The majority of first cannula changes were performed within 3 days after surgery, and loss of airway was recorded in one case. Goldenberg et al. [15] reported on complications related to tracheostomy between 1987 and 1996 in a single center and found eight deaths ( $0.7 \%$ of all tracheostomies) directly related to tracheostomy of which four were due to loss of airway. Our study cannot add data on the specific issue of first cannula changes after tracheostomy, as we focused on long-term tracheotomized patients, and therefore included many patients weeks after tracheostomy has been established in another hospital and a detailed information about the first cannula changes was not available.

To our knowledge, we performed the first study that prospectively correlated patients body measurements as height, weight, neck circumference, and distance between thyroid cartilage and jugulum to the incidence of complications during cannula changes. Interestingly, the measured distance between thyroid cartilage and jugulum was identified as risk factor (OR 1.5, 95\% CI:1.12-2.25, $p=0.02$ ) for patient-threatening complications during cannula changes.

As many patients received tracheostomy in another hospital before being enrolled in this analysis and for various reasons, this study is also not applicable to decide whether patients should be tracheotomized performing PDT or OST. First of all, patients were not randomized between these two techniques, and further information regarding the decision for one or the other procedure is lacking and groups were not equally distributed. We overall observed more cannula changes, more urgencies and more complications in the PDT group compared with patients who had received an OST. However, in logistic regression analysis, no statistical significance could be found. We did not assess other complications related to tracheostomy and its technique, for example surgical site infection, or other possible advantages for one or the other procedure, for example the ability of speech.

There were 79 elective cannula changes in 3191 observation days (2.4/100 observation days) with mechanical complications in $6 \%$ and patient-associated complications in $3 \%$ of them. This is a surprisingly high rate of complications and calls for a further evaluation of indication of these elective tube changes. As we did not expect these findings in this prospective study, no data were assessed regarding the indication of elective changes. However, we conclude that elective changes even in a long-term setting have to be well indicated as they are not without risks. Typical reasons for elective cannula changes were described by White et al. [10], except for certain reasons which can usually not be influenced by the therapeutic team, for example a cuff leak. An experienced team, however, with optimized standards and flow charts may result in less frequent rates of cannula changes in order to change size or length, optimize fitting, or enable speaking.

Interprofessional tracheostomy teams are established and proposed by some authors [5, 6]. It has been reported that an intensivist-led multidisciplinary team is associated with less days of cannulation and length of stay, and that standardized care provided by an interprofessional tracheostomy team is associated with fewer tracheostomy-related complications and an increase in the use of speaking valves [5]. However, a systematic review and a meta-analysis reported that there is only weak evidence supporting the 
statement that tracheostomy teams reduce total tracheostomy time and increase the use of speaking valves [7]. Adding our study to these results, we think that an interprofessional tracheostomy team might be one option to reduce elective and urgent cannula changes, and therefore may lead to fewer patient-associated complications.

One main finding of our study is the influence of time related to the tracheostomy procedure. The need for cannula exchanges and the incidence of $\mathrm{AD}$ is highest during the $3 \mathrm{rd}$ and 4th week after initial tracheostomy, and remains at a high level throughout 8 weeks. These findings underline the extended need for a specialized and well-trained interprofessional care, as well as resources for these specific patients at risk.

\section{Conclusion}

In long-term tracheotomized patients, we found an overall incidence of cannula changes of 4.58 per 100 observation days, and an incidence of urgent cannula changes of 2.1 per 100 tracheostomy days. Mechanical complications occurred in $12 \%$ of cannula changes or 0.56 per 100 tracheostomy days and patient-associated complications in $8 \%$ of cannula changes or 0.34 per 100 tracheostomy days. AD and the requirement of urgent cannula changes for other reasons are common and often related with mechanical and patientassociated complications which are potentially lifethreatening. Even weeks after initial tracheostomy, caregivers have to be aware of serious events and provide monitoring, knowledge, and resources to handle these challenging events.

\section{Data archiving}

The data sets generated and analyzed during this study are available from the corresponding author on reasonable request.

Author contributions UH had full access to all of the data in the study and takes responsibility for the integrity of the data and the accuracy of the data analysis. JW, CW and UH are responsible for the study design. JW, MA, OJ, OK, CW and TS contributed substantially to the study design, data analysis and interpretation, and the writing of the paper.

\section{Compliance with ethical standards}

Conflict of interest The authors declare that they have no conflict of interest.
Ethics This study has been approved by the local ethics committee (Ruhr-University Bochum, Germany, no. of approval 16-5889-BR). We certify that all applicable institutional and governmental regulations concerning the ethical use of human volunteers were followed during the course of this research.

Publisher's note: Springer Nature remains neutral with regard to jurisdictional claims in published maps and institutional affiliations.

\section{References}

1. Durbin CG Jr. Indications for and timing of tracheostomy. Respir Care. 2005;50:483-7.

2. Childs BR, Moore TA, Como JJ, Vallier HA. American spinal injury association impairment scale predicts the need for tracheostomy after cervical spinal cord injury. Spine. 2015;40:1407-13.

3. Heffner JE. Tracheostomy decannulation: marathons and finish lines. Crit Care. 2008;12:128.

4. Wilcox SR, Schmidt UH. Are tracheostomized patients safe on regular hospital wards? Respir Care. 2009;54:1632-4.

5. de Mestral C, Iqbal S, Fong N, LeBlanc J, Fata P, Razek T, et al. Impact of a specialized multidisciplinary tracheostomy team on tracheostomy care in critically ill patients. Can J Surg. 2011;54:167-72.

6. Tobin AE, Santamaria JD. An intensivist-led tracheostomy review team is associated with shorter decannulation time and length of stay: a prospective cohort study. Crit Care. 2008;12: R48.

7. Speed L, Harding KE. Tracheostomy teams reduce total tracheostomy time and increase speaking valve use: a systematic review and meta-analysis. J Crit Care. 2013;28:216 e1-10.

8. Rumbak MJ, Walsh FW, Anderson WM, Rolfe MW, Solomon DA. Significant tracheal obstruction causing failure to wean in patients requiring prolonged mechanical ventilation: a forgotten complication of long-term mechanical ventilation. Chest. 1999;115:1092-5.

9. White AC, Purcell E, Urquhart MB, Joseph B, O'Connor HH. Accidental decannulation following placement of a tracheostomy tube. Respir Care. 2012;57:2019-25.

10. White AC, Kher S, O'Connor HH. When to change a tracheostomy tube. Respir Care. 2010;55:1069-75.

11. Sun GW, Shook TL, Kay GL. Inappropriate use of bivariable analysis to screen risk factors for use in multivariable analysis. $\mathrm{J}$ Clin Epidemiol. 1996;49:907-16.

12. Heinze G, Dunkler D. Five myths about variable selection. Transpl Int. 2017;30:6-10.

13. Tabaee A, Lando T, Rickert S, Stewart MG, Kuhel WI. Practice patterns, safety, and rationale for tracheostomy tube changes: a survey of otolaryngology training programs. Laryngoscope. 2007;117:573-6.

14. Van Buren NC, Narasimhan ER, Curtis JL, Muntz HR, Meier JD. Pediatric tracheostomy: timing of the first tube change. Ann Otol Rhinol Laryngol. 2015;124:374-7.

15. Goldenberg D, Ari EG, Golz A, Danino J, Netzer A, Joachims HZ. Tracheotomy complications: a retrospective study of 1130 cases. Otolaryngol Head Neck Surg. 2000;123:495-500. 